DOI 10.21685/2500-0578-2021-2-3

\title{
SUCCESSIONAL STATE OF FORESTS IN THE UPPER PLATEAU OF THE VOLGA UPLAND
}

\author{
N. A. Leonova \\ Penza State University, 40 Krasnaya street, Penza, 440026, Russia \\ na_leonova@mail.ru
}

Abstract. Background. Long-term and intense anthropogenic impact on the forest-steppe nature of the Russian Plain has led to destruction of vast areas with natural vegetation and significant reduction in their biological diversity. Forests as a component of forest-steppe complexes have undergone the most significant transformation. Materials and methods. The biodiversity analysis was based on geobotanical descriptions obtained as a result of route and stationary studies of forest vegetation. The structural diversity of communities was assessed by the ratio of ecological-coenotic groups (ECGs) of species in the vegetation cover. To assess the successional state, we used the following parameters: representativeness of potential flora, rate of participation of R-species (explents) in the tree layer, dominance degree, proportion of demographically full-member and immoral populations. Results and conclusions. The modern vegetation cover of the upper plateau of the Volga Upland is represented by four formations (pine, birch, aspen, and black alder forests), one group of formations (broad-leaved forests), 34 groups of associations, and 112 associations. It was revealed that the forest flora includes 423 plant species belonging to 257 genera and 85 families. The maximum species richness is noted in the communities of association groups of all the formations dominated by nemoral species. A distinctive feature of the landscape communities of erosion and denudation plains (EDP) is the high constancy of the nemoral species Convallaria majalis in the herbaceous cover. Formation of modern forests is regulated by human. Their transformations are caused by a complex combination of felling of different types and intensity, plowing, grazing, and fires. The existing differences in the succession status of forests of different formations are manifested in changing species composition of stands and floristic diversity of all community synusiae (trees, shrubs, grasses).

Keywords: upper plateau of the Volga Upland, forest vegetation, succession status, floristic diversity, synusia

For citation: Leonova N.A. Successional state of forests in the upper plateau of the Volga Upland. Russian Journal of Ecosystem Ecology. 2021;6(2). Available from: https://doi.org/10.21685/2500-0578-2021-2-3

\section{СУКЦЕССИОННОЕ СОСТОЯНИЕ ЛЕСОВ ВЕРХНЕГО ПЛАТО ПРИВОЛЖСКОЙ ВОЗВЫШЕННОСТИ}

\author{
Н. А. Леонова \\ Пензенский государственный университет, Россия, 440026, г. Пенза, ул. Красная, 40 \\ na_leonova@mail.ru
}

Аннотация. Актуальность и цели. Длительное и интенсивное антропогенное воздействие на природу лесостепи Русской равнины привело к уничтожению огромных площадей естественной растительности, произошло значительное сокращение их биологического разнообразия. Наиболее существенную трансформацию как компонент лесостепных комплексов претерпели леса. Материалы и методы. Изучение лесной растительности осуществлялось при маршрутных и стационарных исследованиях. Полученные геоботанические описания послужили материалом для анализа биоразнообразия. Структурное разнообразие сообществ оценивалось по соотношению в составе растительного покрова эколого-ценотических групп (ЭцГ) видов. Для оценки сукцессионного состояния использовались параметры: представленность потенциальной флоры; доля участия R-видов (эксплерентов) в древесном ярусе; степень доминирования; доля демографически полночленных популяций; доля неморальных. Результаты и выводы. Современный растительный покров верхнего плато Приволжской возвышенности представлен 4 формациями (сосновые, березовые, осиновые, черноольховые леса), 1 группой формаций (широколиственные леса), 34 группами ассоциаций и 112 ассоциациями. В составе флоры лесов выявлено 423 вида растений, принадлежащих к 257 родам и 85 семействам. 
Максимальное видовое богатство отмечено в сообществах групп ассоциаций всех формаций, где наблюдается доминирование неморальных видов. Отличительной особенностью сообществ ландшафтов эрозионноденудационных равнин (ЭДР) является высокое постоянство в травяном покрове неморального вида Convallaria majalis. Современные леса формируются под воздействием человека и трансформированы в результате сложного сочетания рубок разных типов и интенсивности, распашек, выпаса и пожаров. Существующие различия сукцессионного статуса лесов разных формаций проявляются в изменении видового состава древостоев, флористического разнообразия всех синузий (деревьев, кустарников, трав) сообществ.

Ключевые слова: верхнее плато Приволжской возвышенности, лесная растительность, сукцессионный статус, флористическое разнообразие, синузия

Аля цитирования: Леонова Н. А. Сукцессионное состояние лесов верхнего плато Приволжской возвышенности // Russian Journal of Ecosystem Ecology. 2021. Vol. 6 (2). https://doi.org/10.21685/2500-0578-2021-2-3

\section{Introduction}

The study region is located in the central and most developed part of the European Russia and occupies the upper plateau of the Volga Upland (within the boundaries of the Penza region). Large amplitude of heights, readily Paleogene permeable rocks, deep groundwater, widespread loess-like, and solifluction Quaternary deposits contribute to the fact that the main processes of landscape formation in this territory are erosions, suffusions, and, less often, solifluctions. The landscapes of the upper plateau of the Volga Upland are combined into a group of erosion-denudation plain (EDP) landscapes [1-5].

Earlier works [3, 6-10] discussed the species composition, structure, ecological features and confinement of the main types of forests in this area. This work analyzes successional state, dynamics, and development forecast (from a population point of view) of forest communities on the upper plateau of the Volga Upland.

\section{Methods of research}

The forest vegetation on the upper plateau of the Volga Upland is represented by formations of pine, birch, aspen, and black alder forests, as well as by a group of formations of deciduous forests.

Route and stationary studies of the composition and structure of forest vegetation were conducted on test plots (TP) with a size of $10 \mathrm{~m} \mathrm{x} 10 \mathrm{~m}\left(100 \mathrm{~m}^{2}\right)$. The resulting geobotanical descriptions served as material for the analysis of biodiversity.

The structural diversity of communities was assessed by the ratio of ecological-coenotic groups (ECGs) of species, i.e. species ecologically close in their genesis, associated with different types of communities, in the composition of the vegetation cover. We used the ECG classification proposed by O. V. Smirnova and L. B. Zaugolnova [11-13] and refined by the methods of multidimensional statistics by V. E. Smirnov et al. [14] based on the ecological groups proposed by A. A. Nitsenko [15] and taking into account the historical formations proposed by G. M. Zozulin [16, 17]. All vascular plant species noted in geobotanical descriptions were divided into nine ECGs: nemoral $(\mathrm{Nm})$, boreal $(\mathrm{Br})$, nitrophilous $(\mathrm{Nt})$, oligotrophic $(\mathrm{Og})$, piny $(\mathrm{Pn})$, steppe $(\mathrm{St})$, meadow-forest edge $(\mathrm{Md})$, wetland (Wt), and adventive (AdCult).

Within the forest vegetation, formations were grouped by dominants of the tree layer, with the exception of the group of formations of deciduous forests, which was considered as a whole: pine forests with Pinus sylvestris stand as an edificator, aspen forests dominated by Populus tremula, birch forests dominated by Betula pendula, alder (black alder) forests dominated by Alnus glutinosa, and a group of deciduous forest formations dominated by Quercus robur. Groups of associations were identified based on the dominance of ECGs in the grassdwarf shrub layer.

The biodiversity of the vegetation cover was assessed by species saturation on the TP and species (floristic) richness, i.e. the total number of registered species in communities of a certain type, followed by compiling lists of communities of dominant and constant types. Species, for which the numerical value of coverage-abundance on at least one TP included in the description group was at least $40 \%$, were considered dominant. The constancy (constancy class) of the species was calculated according to the following scheme: $1^{\text {st }}$ class the species was present on no more than $20 \%$ of plots in the description group, $2^{\text {nd }}$ class - the species was present on from $20 \%$ to $40 \%$ of plots.

To assess the successional state, we used the following parameters $[11,18-21]$ : representativeness of potential flora - the ratio of the number of species of real flora to the number of species of potential flora in the ecotope (\%); the share of participation of R-species (explents) in the tree layer the ratio of the coverage of explent species to the total coverage of all types of tree canopy (\%); the degree of dominance - the ratio of the coverage of the most abundant species to the total coverage of the species included in the layer (\%); the share of demographically full-member populations the share of species with full-member populations 
in the total number of synusia species (the indicator was calculated for tree and shrub synusiae); the share of nemoral species - the ratio of the total coverage of species of the nemoral group (taking into account the abundance) to the total abundance of grass cover species (\%).

To assess the main ecological parameters, the obtained geobotanical descriptions were processed according to the ecological scales by D. N. Tsyganov [22].

\section{Results and discussion}

The modern vegetation cover in the EDP landscapes is represented by four formations, one group of formations, 34 groups of associations, and 112 associations (Table 1). The communities contain clear traces of economic activity: intensive grazing, felling, recreational load, and fires.

Table 1

Ecological-coenotic classification of EDP forest vegetation

\begin{tabular}{|c|c|}
\hline Association group & Associations \\
\hline 1 & 2 \\
\hline \multicolumn{2}{|c|}{ Pine stand formation - Pineta sylvestris (P) } \\
\hline Pineta boreo-herbosa $(\mathrm{P}-\mathrm{Br})$ & $\begin{array}{l}\text { Pinetum equiseto-moliniosum, Pinetum moliniosum, Pinetum } \\
\text { myrtillosum, Pinetum calamagrostidoso arundinacii-myrtillosum, } \\
\text { Pinetum mixto-herbosum }\end{array}$ \\
\hline $\begin{array}{l}\text { Pineta nemoro-boreo-herbosa } \\
\left(\mathrm{P}-\mathrm{Nm} \_\mathrm{Br}\right)\end{array}$ & $\begin{array}{l}\text { Pinetum calamagrostidosum arundinaceae, Pinetum convallarioso- } \\
\text { calamagrostidosum arundinaceae, Pinetum vaccinioso-hylocomiosum, } \\
\text { Pinetum ruboso saxatili-hylocomiosum, Pinetum mixto-herbosum }\end{array}$ \\
\hline Pineta prato-boreo-herbosa (P-Md_Br) & $\begin{array}{l}\text { Pinetum equsetosum sylvaticum, Pinetum tilieto-herbosum, Pinetum } \\
\text { mixto-herbosum }\end{array}$ \\
\hline $\begin{array}{l}\text { Pineta aqua-uligino-boreo-herbosa } \\
(\mathrm{P}-\mathrm{Wt} \mathrm{Br})\end{array}$ & Pinetum poeosum palustris \\
\hline Pineta nemoro-herbosa $(\mathrm{P}-\mathrm{Nm})$ & $\begin{array}{l}\text { Pinetum coryloso-stellarioso-caricosum pilosae, Pinetum caricosum } \\
\text { pilosae, Pinetum aegopodiosum, Pinetum acerioso-convallariosum, } \\
\text { Pinetum caricosum digitatae, Pinetum convallariosum, Pinetum } \\
\text { stellariosum, Pinetum brachypodioso-convallariosum, Pinetum mixto- } \\
\text { herbosum }\end{array}$ \\
\hline $\begin{array}{l}\text { Pineta boreo-nemoro-herbosa } \\
\left(\mathrm{P}-\mathrm{Br} \_\mathrm{Nm}\right)\end{array}$ & $\begin{array}{l}\text { Pinetum calamagrostidoso-brachypodiosum, Pinetum aceroso- } \\
\text { convallariosum, Pinetum euonymoso-convallariosum, Pinetum } \\
\text { myrtilloso-convallariosum, Pinetum herboso-convallariosum, Pinetum } \\
\text { mixto-herbosum }\end{array}$ \\
\hline Pineta pine-herbosa $(\mathrm{P}-\mathrm{Pn})$ & $\begin{array}{l}\text { Pinetum pteridiosum, Pinetum chamaecytisoso-calamagrostidosum } \\
\text { epigeios, Pinetum calamagrostidosum epigeios }\end{array}$ \\
\hline Pineta boreo-pine-herbosa $\left(\mathrm{P}-\mathrm{Br} \_\mathrm{Pn}\right)$ & $\begin{array}{l}\text { Pinetum herboso-pteridiosum, Pinetum pteridioso-vacciniosum, Pinetum } \\
\text { hylocomiosum, Pinetum herbosum }\end{array}$ \\
\hline Pineta boreo-prato-herbosa $\left(\mathrm{P}-\mathrm{Br} \_\mathrm{Md}\right)$ & Pinetum calamagrostidoso arundinacii-fragariosum \\
\hline $\begin{array}{l}\text { Pineta nemoro-prato-herbosa } \\
\left(\mathrm{P}-\mathrm{Nm} \_\mathrm{Md}\right)\end{array}$ & Pinetum fragariosum, Pinetum agrostidosum, Pinetum mixto-herbosum \\
\hline $\begin{array}{l}\text { Pineta nemoro-pine-herbosa }(\mathrm{P}- \\
\left.\mathrm{Nm} \_\mathrm{Pn}\right)\end{array}$ & $\begin{array}{l}\text { Pinetum herboso-calamagrostidosum epigeios, Pinetum cladinosum, } \\
\text { Pinetum mixto-herbosum }\end{array}$ \\
\hline $\begin{array}{l}\text { Pineta steppe-nemoro-herbosa } \\
\left(\mathrm{P}-\mathrm{St} \_\mathrm{Nm}\right)\end{array}$ & Pinetum coryloso-graminosum, Pinetum aceroso-herbosum \\
\hline Pineta steppe-herbosa $(\mathrm{P}-\mathrm{St})$ & $\begin{array}{l}\text { Pinetum querceto-bromopsiosum ripariae, Pinetum querceto-caricosum } \\
\text { supinae, Pinetum fragarioso-poeosum angustifoliae }\end{array}$ \\
\hline Pineta nitrophilo-herbosa $(\mathrm{P}-\mathrm{Nt})$ & Pinetum urticosum, Pinetum mixto-herbosum \\
\hline \multicolumn{2}{|c|}{ Broad-leaved forest formations - Querceta $(\mathbf{Q})$} \\
\hline Querceta nemoro-herbosa (Q-Nm) & $\begin{array}{l}\text { Quercetum coryloso-caricosum pilosae, Quercetum caricosum pilosae, } \\
\text { Quercetum stellariosum, Quercetum convallariosum, Tilieto-Quercetum } \\
\text { coryloso-aegopodiosum, Acereto-Tilietum coryloso-aegopodiosum, } \\
\text { Aceretum coryloso-aegopodiosum, Quercetum aegopodiosum, } \\
\text { Quercetum brachypodiosum и Quercetum mixto-herbosum. }\end{array}$ \\
\hline $\begin{array}{l}\text { Querceta boreo-nemoro-herbosa } \\
\left(\mathrm{Q}-\mathrm{Br} \_\mathrm{Nm}\right)\end{array}$ & $\begin{array}{l}\text { Quercetum convallarioso-calamagrostidosum arundinacii, Quercetum } \\
\text { rubosum saxatilii }\end{array}$ \\
\hline $\begin{array}{l}\text { Querceta nemoro-pine-herbosa } \\
\text { (Q-Nm_Pn) }\end{array}$ & Quercetum calamagrostidosum epigeios и Quercetum pteridiosum \\
\hline $\begin{array}{l}\text { Querceta nemoro-nitrophilo-herbosa } \\
\text { (Q-Nm_Nt) }\end{array}$ & $\begin{array}{l}\text { Quercetum mixto-herbosum, Quercetum caricosum, Quercetum urticoso- } \\
\text { filipendulosum, Quercetum herboso-urticosum }\end{array}$ \\
\hline
\end{tabular}


End of Table 1

\begin{tabular}{|c|c|}
\hline 1 & 2 \\
\hline $\begin{array}{l}\text { Querceta nemoro-prato-steppe-herbosa } \\
\text { (Q-Nm_Md-St) }\end{array}$ & $\begin{array}{l}\text { Quercetum poeosum angustifoliae, Quercetum bromopsiosum ripariae, } \\
\text { Quercetum herboso-bromopsiosum inermiae, Quercetum caricosum } \\
\text { supinae, Quercetum poeoso angustifoliae-herbosum, Quercetum } \\
\text { fruticoso-herbosum, Quercetum mixto-herbosum. }\end{array}$ \\
\hline \multicolumn{2}{|c|}{ Aspen stand formation - Tremuleta (Pp) } \\
\hline Tremuleta nemoro-herbosa $(\mathrm{Pp}-\mathrm{Nm})$ & $\begin{array}{l}\text { Tremuletum convallariosum, Tremuletum aegopodiosum, Tremuletum } \\
\text { mixto-herbosum, Tremuletum coryloso-caricoso-aegopodiosum, } \\
\text { Tremuletum caricosum pilosae. }\end{array}$ \\
\hline $\begin{array}{l}\text { Tremuleta boreo-nemoro-herbosa } \\
\left(\mathrm{Pp}-\mathrm{Br} \_\mathrm{Nm}\right)\end{array}$ & $\begin{array}{l}\text { Tremuletum calamagrostidosum arundinacae и Tremuletum mixto- } \\
\text { herbosum }\end{array}$ \\
\hline $\begin{array}{l}\text { Tremuleta nemoro-pine-herbosa } \\
\text { (Pp-Nm_Pn) }\end{array}$ & Tremuletum pteridiosum \\
\hline $\begin{array}{l}\text { Tremuleta prato-nemoro-herbosa } \\
\text { (Pp-Md_Nm) }\end{array}$ & $\begin{array}{l}\text { Tremuletum bromopsioso-brachypodiosum, Tremuletum } \\
\text { brachypodiosum и Tremuletum mixto-herbosum }\end{array}$ \\
\hline $\begin{array}{l}\text { Tremuleta nitrophilo-nemoro-herbosa } \\
\text { (Pp-Nt_Nm) }\end{array}$ & Tremuletum mixto-herbosum \\
\hline \multicolumn{2}{|c|}{ Birch stand formation - Betuleta $(\mathrm{B})$} \\
\hline Betuleta nemoro-herbosa (B-Nm) & $\begin{array}{l}\text { Betuletum caricosum pilosae, Betuletum aegopodioso-caricosum pilosae, } \\
\text { Betuletum aegopodiosum, Betuletum melampyrosum, Betuletum } \\
\text { convallariosum, Betuletum brachypodiosum, Betuletum caricosum } \\
\text { digitatae, Betuletum mixto-herbosum }\end{array}$ \\
\hline $\begin{array}{l}\text { Betuleta nemoro-boreo-herbosa } \\
\text { (B- Nm_Br) }\end{array}$ & $\begin{array}{l}\text { Betuletum aegopodioso- calamagrostidosum arundinacae, Betuletum } \\
\text { herboso-calamagrostidosum arundinacae и Betuletum mixto-herbosum }\end{array}$ \\
\hline Betuleta boreo-herbosa (B-Br) & $\begin{array}{l}\text { Betuletum rubosum saxatilis, Betuletum mixto-herbosum, Betuletum } \\
\text { pyrolosum rotundifoliae, Betuletum molinioso-myrtillsum, Betuletum } \\
\text { myrtillosum и Betuletum moliniosum }\end{array}$ \\
\hline $\begin{array}{l}\text { Betuleta nemoro-pine-herbosa } \\
\text { (B-Nm_Pn) }\end{array}$ & $\begin{array}{l}\text { Betuletum calamagrostidoso-pteridiosum, Betuletum hylocomiosum, } \\
\text { Betuletum calamagrostidosum epigeios u Betuletum pteridiosum }\end{array}$ \\
\hline Betuleta prato-herbosa (B-Md) & Betuletum poeoso angustifolii-fragariosum \\
\hline Betuleta nitrophilo-herbosa $(\mathrm{B}-\mathrm{Nt})$ & Betuletum urticosum и Betuletum mixto-herbosum \\
\hline $\begin{array}{l}\text { Betuleta boreo-aqua-uligino-herbosa } \\
\text { (B-Br_Wt) }\end{array}$ & Betuletum calamagrostidosum canescens \\
\hline \multicolumn{2}{|c|}{ Black alder stand formation - Alneta glutinosa (A) } \\
\hline Alneta nitrophilo-herbosa (A-Nt) & $\begin{array}{l}\text { Alnetum herboso-aegopodiosoum, Alnetum aegopodioso-urticosum, } \\
\text { Alnetum herboso-urticosum }\end{array}$ \\
\hline $\begin{array}{l}\text { Alneta nemoro-nitrophilo-herbosa } \\
\left(\mathrm{A}-\mathrm{Nm} \_\mathrm{Nt}\right)\end{array}$ & Alnetum athyriosum \\
\hline $\begin{array}{l}\text { Alneta nitrophilo-aqua-uligino-herbosa } \\
\text { (A-Nt_Wt) }\end{array}$ & Alnetum caricosum riparii \\
\hline
\end{tabular}

We revealed that the forest flora includes 423 plant species belonging to 257 genera and 85 families, among which dicotyledonous flowering plants predominate.

The maximum species richness was noted in the communities of the groups of associations of all the formations with the dominance of nemoral species, while the total number of species in deciduous forests with nemoral grasses is two or more times more than in other groups of associations (Table 2).

Table 2

The number of plant species in landscapes of erosion-denudation plains

\begin{tabular}{|c|c|c|c|c|c|c|c|}
\hline \multirow{2}{*}{ Formation } & \multirow{2}{*}{$\begin{array}{l}\text { Association } \\
\text { groups }\end{array}$} & \multirow{2}{*}{$\begin{array}{c}\text { Average species } \\
\text { saturation }\end{array}$} & \multicolumn{5}{|c|}{ Number of species: } \\
\hline & & & general & trees & shrubs & grasses & mosses and lichens \\
\hline 1 & 2 & 3 & 4 & 5 & 6 & 7 & 8 \\
\hline \multirow{7}{*}{$\mathrm{P}$} & $\mathrm{P}-\mathrm{Br}$ & 16,3 & 99 & 10 & 10 & 74 & 5 \\
\hline & $\mathrm{P}-\mathrm{Nm} \_\mathrm{Br}$ & 19,1 & 138 & 12 & 10 & 110 & 6 \\
\hline & P-Md_Br & 15,5 & 92 & 10 & 7 & 75 & \\
\hline & P-Wt_Br & 16,5 & 24 & 2 & 1 & 21 & \\
\hline & $\mathrm{P}-\mathrm{Nm}$ & 14,2 & 126 & 11 & 9 & 104 & 2 \\
\hline & P-Br_Nm & 16,8 & 161 & 14 & 11 & 130 & 6 \\
\hline & P-Pn & 16,2 & 92 & 10 & 9 & 69 & 4 \\
\hline
\end{tabular}


End of Table 2

\begin{tabular}{|c|c|c|c|c|c|c|c|}
\hline 1 & 2 & 3 & 4 & 5 & 6 & 7 & 8 \\
\hline \multirow{7}{*}{$\mathrm{P}$} & P-Br_Md & 22,1 & 44 & 5 & 3 & 33 & 3 \\
\hline & P-Br_Pn & 19,4 & 121 & 13 & 9 & 93 & 6 \\
\hline & P-St_Nm & 13,8 & 58 & 10 & 6 & 42 & \\
\hline & P-Nm_Md & 15,5 & 104 & 10 & 7 & 85 & 2 \\
\hline & P-Nm_Pn & 17,6 & 128 & 9 & 9 & 104 & 6 \\
\hline & $\mathrm{P}-\mathrm{St}$ & 17,5 & 74 & 6 & 5 & 63 & \\
\hline & $\mathrm{P}-\mathrm{Nt}$ & 12,3 & 55 & 12 & 9 & 34 & \\
\hline \multirow{5}{*}{$\mathrm{Q}$} & Q-Nm & 13,7 & 216 & 15 & 9 & 191 & 1 \\
\hline & Q-Br_Nm & 14,1 & 60 & 9 & 3 & 48 & \\
\hline & Q- Nm_Md_St & 15,3 & 198 & 12 & 13 & 173 & \\
\hline & Q-Nm_Nt & 12,4 & 95 & 13 & 9 & 73 & \\
\hline & Q-Nm_Pn & 16,6 & 97 & 10 & 3 & 84 & \\
\hline \multirow{5}{*}{$\mathrm{Pp}$} & $\mathrm{Pp}-\mathrm{Nm}$ & 10,7 & 133 & 11 & 6 & 113 & 3 \\
\hline & Pp-Br_Nm & 12,4 & 92 & 10 & 9 & 71 & 2 \\
\hline & Pp-Nm_Pn & 15,8 & 64 & 7 & 3 & 54 & \\
\hline & Pp-Md_Nm & 17,5 & 86 & 10 & 6 & 70 & \\
\hline & $\mathrm{Pp}-\mathrm{Nt} \_\mathrm{Nm}$ & 9,1 & 20 & 6 & & 14 & \\
\hline \multirow{7}{*}{ B } & B-Nm & 12,6 & 186 & 14 & 10 & 159 & 3 \\
\hline & B-Nm_Br & 13,7 & 75 & 9 & 7 & 56 & 3 \\
\hline & $\mathrm{B}-\mathrm{Br}$ & 14,8 & 113 & 11 & 6 & 92 & 4 \\
\hline & B-Nm_Pn & 19,6 & 148 & 9 & 9 & 124 & 6 \\
\hline & B-Md & 26,7 & 46 & 4 & & 42 & \\
\hline & $\mathrm{B}-\mathrm{Nt}$ & 8,8 & 24 & 5 & 3 & 16 & \\
\hline & B-Br_Wt & 22,3 & 49 & 3 & 1 & 45 & 1 \\
\hline \multirow{3}{*}{$\mathrm{A}$} & A-Nt_Wt & 26,3 & 50 & 5 & 1 & 42 & 2 \\
\hline & $\mathrm{A}-\mathrm{Nt}$ & 21,4 & 41 & 6 & 1 & 32 & 2 \\
\hline & A-Nm_Nt & 24,2 & 86 & 9 & 4 & 71 & 2 \\
\hline
\end{tabular}

Nemoral broad-leaved forests are leaders in terms of the number of species and the species richness of tree and grass synusiae. In second place are deciduous forests with a predominance of species of nemoral, meadow, and steppe ECGs. These communities are characterized by the highest abundance of shrub synusia. Among the formation of pine forests, the maximum species richness is characteristic of communities with a high participation of boreal and nemoral species. Low values of species richness are typical for black alder forests and forests with a high proportion of nitrophilous and wetland ECG species in the herbaceous layer.

The total species richness in the noted communities varies from 9.1 to 26.7 species per $100 \mathrm{~m}^{2}$. The lowest value of the total species saturation is typical for nitrophilous birch forests, which is obviously explained by strong anthropogenic transformation of the vegetation of this group of associations and absolute dominance of Urtica dioica in the herbaceous layer that leads to the loss of species diversity.
The communities of most groups of associations are characterized by slightly different values of species saturation (10.7-19.6). The highest rates were recorded in meadow birch forests -26.7 forests and nitrophytic wetland black alder forests forests and nitrophytic wetland black alder forests forests and nitrophytic wetland black alder forests 26.3 species per $100 \mathrm{~m}^{2}$. They were due to the introduction of a large number of meadow-steppe species to the formers and nitrophilous and wetland species to the latters.

The analysis of the flora of the identified formations showed (Table 3 ) a high vegetation similarity of pine and birch forests, a medium similarity of aspen forests and birch and pine forests, as well as similarity of broad-leaved forests and birch forests, aspen forests and pine forests, which is obviously explained by the similar participation in their species composition of nemoral, meadow, and boreal ECGs as well as similar highly constant species (Table 4).

Table 3

Floristic similarity of formations in EDP landscapes (Jaccard coefficient)

\begin{tabular}{|c|c|c|c|c|c|}
\hline Formation & B & P & Pp & Q & A \\
\hline B & & 0.64 & 0.51 & 0.53 & 0.20 \\
\hline P & & & 0.49 & 0.56 & 0.19 \\
\hline $\mathrm{Pp}$ & & & & 0.49 & 0.15 \\
\hline $\mathrm{Q}$ & & & & & 0.17 \\
\hline
\end{tabular}


High-constant types of association groups ( $4^{\text {th }}$ and $5^{\text {th }}$ constancy classes)

\begin{tabular}{|c|c|c|c|c|c|c|c|c|c|c|c|c|c|c|c|c|c|c|c|c|c|c|c|c|c|c|c|c|c|c|c|c|c|c|}
\hline \multirow[t]{3}{*}{ Formation } & \multicolumn{14}{|c|}{$\mathrm{P}$} & \multicolumn{5}{|c|}{$\mathrm{Q}$} & \multicolumn{5}{|c|}{ Pp } & \multicolumn{7}{|c|}{$\mathrm{B}$} & \multicolumn{3}{|c|}{ A } \\
\hline & & & & & & & & & & & & & & & & $\mathrm{ssO}$ & ciati & ion & grol & & & & & & & & & & & & & & & \\
\hline & 穴 & $\begin{array}{l}\bar{n} \\
\xi \\
\bar{z}\end{array}$ & $\begin{array}{l}\vec{n} \\
\vec{\Sigma} \\
\vec{\Sigma}\end{array}$ & $\mid \begin{array}{l}\vec{n} \\
\overrightarrow{3} \\
\overrightarrow{3}\end{array}$ & $\xi$ & $\mid \begin{array}{l}\tilde{z} \\
\dot{\bar{n}} \\
\dot{1}\end{array}$ & $\approx$ & $\mid \begin{array}{l}\overrightarrow{\Sigma_{1}} \\
\bar{\omega}_{1}\end{array}$ & $\begin{array}{c}\vec{n} \\
\dot{n} \\
\dot{n}\end{array}$ & $\left|\begin{array}{c}\Xi \\
\vec{z}_{1} \\
\dot{\omega}\end{array}\right|$ & $\mid \begin{array}{l}\tilde{z} \\
\Sigma \\
z\end{array}$ & $\mid \begin{array}{l}\tilde{D} \\
\xi_{1} \\
z\end{array}$ & $\vec{n}$ & $\vec{z}$ & $\xi$ & $\begin{array}{l}\xi \\
z_{1} \\
\dot{n}\end{array}$ & $\mid \begin{array}{l}\vec{z}_{1} \\
\Xi_{1} \\
\bar{z}\end{array}$ & $\begin{array}{l}\vec{z}_{1} \\
z_{z} \\
z_{1}\end{array}$ & $\begin{array}{l}\tilde{z} \\
\xi \\
z\end{array}$ & $\xi$ & $\mid \begin{array}{l}\xi \\
z_{1} \\
\ddot{n}\end{array}$ & $\left|\begin{array}{c}z \\
2 \\
\vdots \\
z \\
z\end{array}\right|$ & $\begin{array}{l}\Xi_{2} \\
\vec{z}_{1}\end{array}$ & $\begin{array}{l}\xi_{2} \\
z_{1} \\
\vec{z}^{\prime}\end{array}$ & $\Xi$ & $\begin{array}{l}\overline{\hat{n}_{1}} \\
\bar{z} \\
\bar{z}\end{array}$ & $\dot{\infty}$ & $\left|\begin{array}{l}\tilde{2} \\
\vdots \\
z \\
z\end{array}\right|$ & $\vec{\Sigma}$ & $\vec{z}$ & $\left|\begin{array}{l}3 \\
-1 \\
-1\end{array}\right|$ & $\mid \begin{array}{l}\hat{z}_{1} \\
\vec{z}^{\prime}\end{array}$ & $\vec{z}$ & \begin{tabular}{|l}
$\bar{z}$ \\
$\Xi$ \\
$z$
\end{tabular} \\
\hline 1 & 2 & 3 & 4 & 5 & 6 & 7 & 8 & \begin{tabular}{|l|}
9 \\
\end{tabular} & 10 & 11 & 12 & 13 & 14 & 15 & 16 & 17 & 18 & 19 & 20 & 21 & 22 & 23 & 24 & 25 & 26 & 27 & 28 & 29 & 30 & 31 & 32 & 33 & 34 & 35 \\
\hline $\begin{array}{l}\text { Convallaria } \\
\text { majalis }\end{array}$ & IV & V & IV & $\mathrm{V}$ & & V & V & $\mathrm{V}$ & $\mathrm{V}$ & $\mathrm{V}$ & & $\mathrm{V}$ & & & & V & & & IV & IV & $\mathrm{V}$ & IV & & $\mathrm{V}$ & IV & & & IV & & & & & & \\
\hline $\begin{array}{l}\text { Sorbus } \\
\text { aucuparia }\end{array}$ & IV & & & $\mathrm{V}$ & & IV & $\mathrm{V}$ & $\mathrm{V}$ & V & $\mathrm{V}$ & IV & & & & & & & & & & IV & & & & & $\mathrm{V}$ & IV & V & & & IV & & $\mathrm{V}$ & IV \\
\hline $\begin{array}{l}\text { Pinus } \\
\text { sylvestris }\end{array}$ & V & V & $\mathrm{V}$ & $\mathrm{V}$ & V & V & V & $\mathrm{V}$ & $\mathrm{V}$ & $\mathrm{V}$ & V & $\mathrm{V}$ & $\mathrm{V}$ & V & & & & & & & & & & & & & & & & & & & & \\
\hline Betula pendula & IV & & & & & & & $\mathrm{V}$ & & & & & & IV & & & & & & & & & $\mathrm{V}$ & & $\mathrm{V}$ & $\mathrm{V}$ & $\mathrm{V}$ & $\mathrm{V}$ & & $\mathrm{V}$ & & & & \\
\hline Quercus robur & & 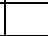 & & & IV & & & & & & V & & IV & & $\mathrm{V}$ & $\mathrm{V}$ & $\mathrm{V}$ & $\mathrm{V}$ & V & & 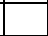 & & IV & & & 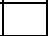 & & . & & & & & & \\
\hline $\begin{array}{l}\text { Calamagrostis } \\
\text { arundinacea }\end{array}$ & IV & IV & 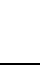 & & & IV & & $\mathrm{V}$ & & & & & & & & IV & & & & & IV & & & & & $\mathrm{V}$ & & IV & & & & & & \\
\hline Fragaria vesca & & $\mathrm{V}$ & $\mathrm{V}$ & & & & & $\mathrm{V}$ & & & IV & & IV & & & & & & IV & & & & IV & & & 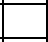 & & & $\mathrm{V}$ & & & & & \\
\hline $\begin{array}{l}\text { Populus } \\
\text { tremula }\end{array}$ & & & & & & & & & & & & & & & & 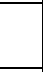 & & & & $\mathrm{V}$ & $\mathrm{V}$ & $\mathrm{V} \mid$ & V & $\mathrm{V}$ & & $\mathrm{V}$ & & IV & & & & & & \\
\hline $\begin{array}{l}\text { Calamagrostis } \\
\text { epigeios }\end{array}$ & & & & & & & $\mathrm{V}$ & & & IV & & $\mathrm{IV}$ & & & & & & & IV & & & & & & & & & IV & IV & & & & & \\
\hline $\begin{array}{l}\text { Stachys } \\
\text { officinalis }\end{array}$ & & & & & & & & IV & & & & & & & & IV & IV & & IV & & & & IV & & & & & & IV & & & & & \\
\hline $\begin{array}{l}\text { Aegopodium } \\
\text { podagraria }\end{array}$ & & & & & & & & & & & & & & & IV & & & & & IV & & & & & & IV & & & & & & IV & & V \\
\hline $\begin{array}{l}\text { Brachypodium } \\
\text { pinnatum }\end{array}$ & & & & & & & & & & & IV & & & & & $\mathrm{V}$ & & & V & & & IV & & & & & & & IV & & & & & \\
\hline $\begin{array}{l}\text { Euonymus } \\
\text { verrucosa }\end{array}$ & & & & & & & & & & & & & & & & V & & & IV & IV & & & & & IV & $\mathrm{V}$ & & & & & & & & \\
\hline Padus avium & & & & & & & & & & & & & & V & & & & $\mathrm{V}$ & & & & & & $\mathrm{V}$ & & & & & 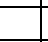 & & & $\mathrm{V}$ & & V \\
\hline $\begin{array}{l}\text { Poa } \\
\text { angustifolia }\end{array}$ & & & & & & & & & & IV & & & $\mathrm{V}$ & & & & IV & & IV & & & & & & & & & & V & & & & & \\
\hline Rubus saxatilis & IV & & & & & & & $\mathrm{V}$ & & & & & & & & & & & & & & & & & & $\mathrm{V}$ & $\mathrm{V}$ & & & & $\mathrm{V}$ & & & \\
\hline $\begin{array}{l}\text { Glechoma } \\
\text { hederacea }\end{array}$ & & & & & & & & & & & & & & & & & & IV & & & & & & & & & & & & & & IV & $\mathrm{V}$ & V \\
\hline $\begin{array}{l}\begin{array}{l}\text { Alnus } \\
\text { glutinosa }\end{array} \\
\end{array}$ & & & & & & & & & & & & & & & & & & & & & & & & & & & & & & & & $\mathrm{V}$ & $\mathrm{V}$ & V \\
\hline $\begin{array}{l}\text { Filipendula } \\
\text { ulmaria }\end{array}$ & & & & & & & & & & & & & & & & & & & & & & & & & & & & & & & & $\mathrm{V}$ & $\mathrm{V}$ & IV \\
\hline $\begin{array}{l}\text { Lysimachia } \\
\text { vulgaris }\end{array}$ & & & & & & & & & & & & & & & & & & & & & & & & & & & & & & IV & $\mathrm{V}$ & $\mathrm{V}$ & & \\
\hline Melica nutans & & IV & & & & & & & & IV & & & & & & & & & & & & & & & & & & & & & & & & \\
\hline $\begin{array}{l}\text { Pleurozium } \\
\text { schreberi }\end{array}$ & & V & & & & & & $\mathrm{V}$ & IV & & & & & & & & & & & & & & & & & & & & & & & & & \\
\hline $\begin{array}{l}\text { Pyrethrum } \\
\text { corymbosum }\end{array}$ & & & & & & & & & & & & & IV & & & & & & IV & & & & & & & & & & V & & & & & \\
\hline Tilia cordata & & & & & IV & & & & & & & & & & & & & & & & & & & & $\mathrm{V}$ & $\mathrm{V}$ & & & & & & & & \\
\hline $\begin{array}{l}\text { Dryopteris } \\
\text { carthusiana }\end{array}$ & & & & & & & & & & & & & & & & & & & & & & & & & & & & & & & & IV & $\mathrm{V}$ & \\
\hline Frangula alnus & & & & & & & & & & & & & & & & & & & & & & & & & & & & & & $\mathrm{V}$ & & $\mathrm{V}$ & & \\
\hline $\begin{array}{l}\text { Geum } \\
\text { urbanum }\end{array}$ & & & & & & & & & & & & & & IV & & & & & & & & & & IV & & & & & & & & & & \\
\hline $\begin{array}{l}\text { Hieracium } \\
\text { umbellatum }\end{array}$ & & & & & & & V & & IV & & & & & & & & & & & & & & & & & & & & & & & & & \\
\hline $\begin{array}{l}\text { Maianthemum } \\
\text { bifolium }\end{array}$ & & & & $\mathrm{V}$ & & & & & & & & & & & & & & & & & & & & & & & & & & & $\mathrm{V}$ & & & \\
\hline $\begin{array}{l}\text { Potentilla } \\
\text { erecta }\end{array}$ & & & & $\mathrm{V}$ & & & & & & & & & & & & & & & & & & & & & & & & & & & $\mathrm{V}$ & & & \\
\hline $\begin{array}{l}\text { Scirpus } \\
\text { sylvaticus }\end{array}$ & & V & & & & & & & & & & & & & & & & & & & & & & & & & & & & $\mathrm{V}$ & & & & \\
\hline $\begin{array}{l}\text { Stellaria } \\
\text { holostea }\end{array}$ & & & & & & & & & & IV & & & & & & & & & & & & & & & & & & & IV & & & & & \\
\hline $\begin{array}{l}\text { Trifolium } \\
\text { alpestre }\end{array}$ & & & & & & & & & & & & & IV & & & & & $\mathrm{V}$ & & & & & & & & & & & V & & & & & \\
\hline Urtica dioica & & & & & & & & & & & & & & & & & & $\mathrm{V}$ & & & & & & & & & & & & & & & & $\mathrm{V}$ \\
\hline Ulmus glabra & & & & & & & & & & & & & & & & & & & & & & & & & & & & & $\mathrm{V}$ & & IV & & & \\
\hline $\begin{array}{l}\text { Acer } \\
\text { platanoides }\end{array}$ & & & & & & & & & & & & & & & & & & & & & & & & & & IV & & & & & & & & \\
\hline $\begin{array}{l}\text { Achillea } \\
\text { millefolium }\end{array}$ & & & & & & & & & & & & & & & & & & & & & & & & & & & & & IV & & & & & \\
\hline
\end{tabular}


End of Table 4

\begin{tabular}{|c|c|c|c|c|c|c|c|c|c|c|c|c|c|c|c|c|c|c|c|c|c|c|c|c|c|c|c|c|c|c|c|c|c|c|}
\hline 1 & 2 & 3 & 4 & 5 & 6 & 7 & 8 & 9 & 10 & 11 & 12 & 13 & 14 & 15 & \begin{tabular}{|l|}
16 \\
\end{tabular} & 17 & 18 & \begin{tabular}{|l|}
19 \\
\end{tabular} & 20 & 21 & 22 & 23 & 24 & 25 & 26 & 27 & 28 & 29 & 30 & 31 & 32 & 33 & 34 & 35 \\
\hline $\begin{array}{l}\text { Antennaria } \\
\text { dioica }\end{array}$ & & & & & & & & IV & & & & & & & & & & & & & & & & & & & & & & & & & & \\
\hline \begin{tabular}{|l} 
Athyrium \\
filix-femina
\end{tabular} & & & & & & & & & & & & & & & & & & & & & & & & & & & & & & & & & V & \\
\hline $\begin{array}{l}\text { Bromopsis } \\
\text { riparia }\end{array}$ & & & & & & & & & & & & & & & & & IV & & & & & & & & & & & & & & & & & \\
\hline $\begin{array}{l}\text { Chamaecytisus } \\
\text { ruthenicus }\end{array}$ & & & & & & & & & & & & IV & & & & & & & & & & & & & & & & & & & & & & \\
\hline $\begin{array}{l}\text { Calamagrostis } \\
\text { canescens }\end{array}$ & & & & & & & & & & & & & & & & & & & & & & & & & & & & & & & & & $\mathrm{V}$ & \\
\hline \begin{tabular}{|l|} 
Carex \\
ericetorum
\end{tabular} & & & & & & & & V & & & & & & & & & & & & & & & & & & & & & & & & & & \\
\hline Carex riparia & & & & & & & & & & & & & & & & & & & & & & & & & & & & & & & & $\mathrm{V}$ & & \\
\hline Carex vaginata & & & & $\mathrm{V}$ & & & & & & & & & & & & & & & & & & & & & & & & & & & & & & \\
\hline \begin{tabular}{|l|}
$\begin{array}{l}\text { Dactylis } \\
\text { glomerata }\end{array}$ \\
\end{tabular} & & & & & & & & & & & & & & & & & & & & & & & & & & & & & V & & & & & \\
\hline $\begin{array}{l}\text { Deschampsia } \\
\text { cespitosa }\end{array}$ & & & & & & & & & & & & & & & & & & & & & & & & & & & & & & & & V & & \\
\hline \begin{tabular}{|l|} 
Dicranum \\
scoparium
\end{tabular} & & & & & & & & V & & & & & & & & & & & & & & & & & & & & & & & & & & \\
\hline $\begin{array}{l}\text { Dryopteris } \\
\text { cristata }\end{array}$ & & & & & & & & & & & & & & & & & & & & & & & & & & & & & & & & IV & & \\
\hline $\begin{array}{l}\text { Equisetum } \\
\text { sylvaticum }\end{array}$ & & & & $\mathrm{V}$ & & & & & & & & & & & & & & & & & & & & & & & & & & & & & & \\
\hline $\begin{array}{l}\text { Hieracium } \\
\text { pilosella }\end{array}$ & & & & & & & & & & & & & & & & & & & & & & & & & & & & & $\mathrm{V}$ & & & & & \\
\hline $\begin{array}{l}\text { Humulus } \\
\text { lupulus }\end{array}$ & & & & & & & & & & & & & & & & & & & & & & & & & & & & & & V & & & & \\
\hline $\begin{array}{l}\text { Lathyrus } \\
\text { vernus }\end{array}$ & & & & & & & & & & & & & & & & & & & & & & & & & & & & & IV & & & & & \\
\hline $\begin{array}{l}\text { Leucanthemum } \\
\text { vulgare }\end{array}$ & & & & & & & & & & & & & & & & & & & & & & & & & & & & & $\mathrm{V}$ & & & & & \\
\hline $\begin{array}{l}\begin{array}{l}\text { Lupinaster } \\
\text { pentaphyllus }\end{array} \\
\end{array}$ & & & & & & & & IV & & & & & & & & & & & & & & & & & & & & & & & & & & \\
\hline $\begin{array}{l}\text { Lycopus } \\
\text { europaeus }\end{array}$ & & & & & & & & & & & & & & & & & & & & & & & & & & & & & & & & $\mathrm{V}$ & & \\
\hline $\begin{array}{l}\text { Melampyrum } \\
\text { pratense }\end{array}$ & & IV & & & & & & & & & & & & & & & & & & & & & & & & & & & & & & & & \\
\hline $\begin{array}{l}\text { Molinia } \\
\text { caerulea }\end{array}$ & & & & $\mathrm{V}$ & & & & & & & & & & & & & & & & & & & & & & & & & & & & & & \\
\hline $\begin{array}{l}\text { Orthilia } \\
\text { secunda }\end{array}$ & & IV & & & & & & & & & & & & & & & & & & & & & & & & & & & & & & & & \\
\hline $\begin{array}{l}\text { Phragmites } \\
\text { australis }\end{array}$ & & & & & & & & & & & & & & & & & & & & & & & & & & & & & & & & & V & \\
\hline $\begin{array}{l}\text { Pimpinella } \\
\text { saxifraga }\end{array}$ & & & & & & & & & & & & & & & & & & & & & & & & & & & & & V & & & & & \\
\hline Poa palustris & & & & $\mathrm{V}$ & & & & & & & & & & & & & & & & & & & & & & & & & & & & & & \\
\hline Potentilla alba & & & & & & & & & & & & & & & & & & & & & & & & & & & & & IV & & & & & \\
\hline $\begin{array}{l}\text { Potentilla } \\
\text { argentea }\end{array}$ & & & & & & & & & & & & & & & & & & & & & & & & & & & & & IV & & & & & \\
\hline $\begin{array}{l}\text { Pteridium } \\
\text { aquilinum }\end{array}$ & & & & & & & & & & & & & & & & & & & & & & V & & & & & & & & & & & & \\
\hline $\begin{array}{l}\text { Pulmonaria } \\
\text { obscura }\end{array}$ & & & & & & & & & & & & & & & & & & & & & & & & & & IV & & & & & & & & \\
\hline $\begin{array}{l}\text { Pulsatilla } \\
\text { patens }\end{array}$ & & & & & & & & V & & & & & & & & & & & & & & & & & & & & & & & & & & \\
\hline $\begin{array}{l}\text { Pyrola } \\
\text { rotundifolia }\end{array}$ & & & & & & & & & & & & & & & & & & & & & & & & & & & & & & & V & & & \\
\hline Ribes nigrum & & & & & & & & & & & & & & & & & & & & & & & & & & & & & & IV & & & & \\
\hline Silene viscosa & & & & & & & & IV & & & & & & & & & & & & & & & & & & & & & & & & & & \\
\hline $\begin{array}{l}\text { Solidago } \\
\text { virgaurea }\end{array}$ & & & & & & & & V & & & & & & & & & & & & & & & & & & & & & & & & & & \\
\hline \begin{tabular}{|l} 
Trientalis \\
europaea
\end{tabular} & & & & & & & & & & & & & & & & & & & & & & & & & & & & & & & $\mathrm{V}$ & & & \\
\hline Ulmus glabra & & & & & & & & & & & & & & & & & & IV & & & & & & & & & & & & & & & & \\
\hline $\begin{array}{l}\text { Vaccinium } \\
\text { myrtillus } \\
\end{array}$ & IV & & & & & & & & & & & & & & & & & & & & & & & & & & & & & & & & & \\
\hline \begin{tabular}{|l|} 
Vaccinium \\
vitis-idaea \\
\end{tabular} & & IV & & & & & & & & & & & & & & & & & & & & & & & & & & & & & & & & \\
\hline Urtica dioic & & & & & & & & & & & & & & & & & & & & & & & & & & & & & & & $\mathrm{V}$ & & & \\
\hline
\end{tabular}

Note: The species with the highest occurrence in forests and erosion-denudation plain landscapes are highlighted in bold and color. 
The phytocenoses are characterized by 74 highly constant species (see Table 4). A distinctive feature of the EDP communities is the high constancy of the nemoral species in the grass cover-Convallaria majalis.
Differences in the composition of communities are also expressed in quantitative relationships between different ECGs (Fig. 1). A high participation of species of nemoral, meadow, and boreal ECGs is quite clearly traced in almost all groups of associations.

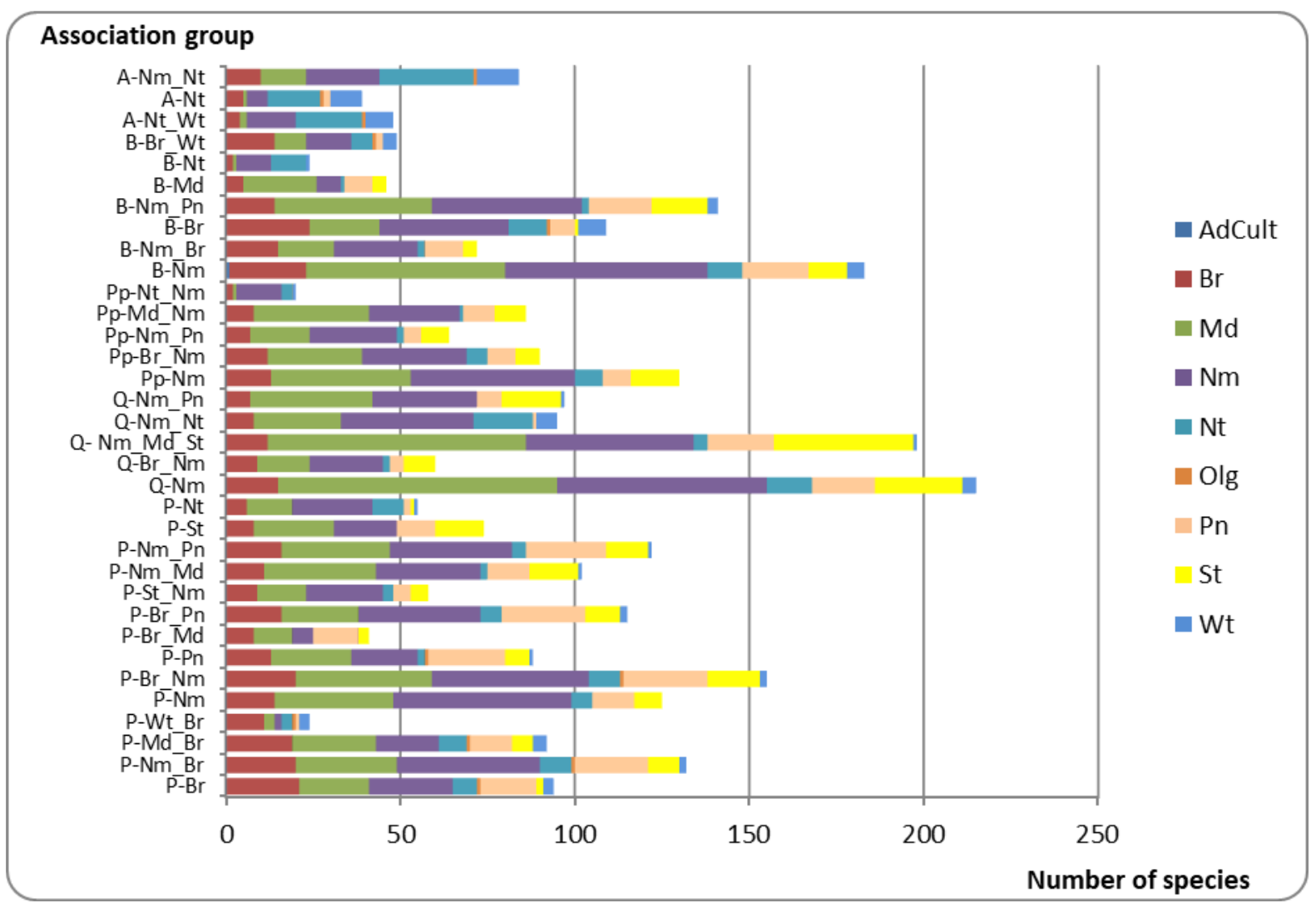

Fig. 1. The number of species of different ecological-coenotic groups in the flora of association groups in erosion-denudation plain landscapes

The ecological analysis of geobotanical descriptions showed the greatest correlation of different groups of associations with soil characteristics such as moisture and nitrogen content, generalized salt regime, and acidity (Fig. 2). Communities of different formations with a high participation of meadow-steppe species occupy the driest, the richest in peat, poor in nitrogen, and close to neutral habitats. The best soils for forests dominated by boreal ECG species are the most humid, acidic, but poor in terms of the generalized salt regime and nitrogen.

Communities with a high participation of nitrophilous ECG species occupy well-moisturized and nitrogen-rich habitats with weakly acidic soils. The ecological space of groups of associations with a predominance of species of nemoral ECGs have similar scoring indicators and mutually intersect for most environmental factors, while, according to the generalized salt regime factor, nemoral deciduous forests occupy the richest habitats in terms of this environmental factor. Nemoral pine, birch, and aspen forests are characterized by similar lower soil peat content, but differ from each other in soil moisture content - birch forests occupy the least moisturized soils, whereas aspen forests occupy the wettest soils

The lightest forests are communities with a predominance of meadow-steppe species; they are also characterized by moderately variable soil moisture content. Nemoral deciduous forests are the most shaded forests. Forests with a high proportion of boreal species are characterized by a medium illumination score, but they occupy habitats with relatively stable moisture content.

The assessment of the successional state of the forest cover of the EDP landscapes showed (Table 5, Fig. 3) that all modern forests are strongly transformed and are at different demutation stages after felling of varying degrees of intensity, grazing, fires, and plowing.

In general, all forests are characterized by low preservation rate of ontogenetic abundance of arboreal synusia and floristic diversity in almost all synusiae (see Fig. 3). Approximately $80 \%$ of the area of EDP landscapes is occupied by phytocenoses, in which the forest stand is formed by $\mathrm{R}$-species, which are explents. The highest onto- 
genetic abundance of trees were noted in nemoral birch and nemoral pine forests. This is obviously due to the fact that a significant number of trees can recover from stumps as shrubs under favorable light conditions that develop in these types of forests.

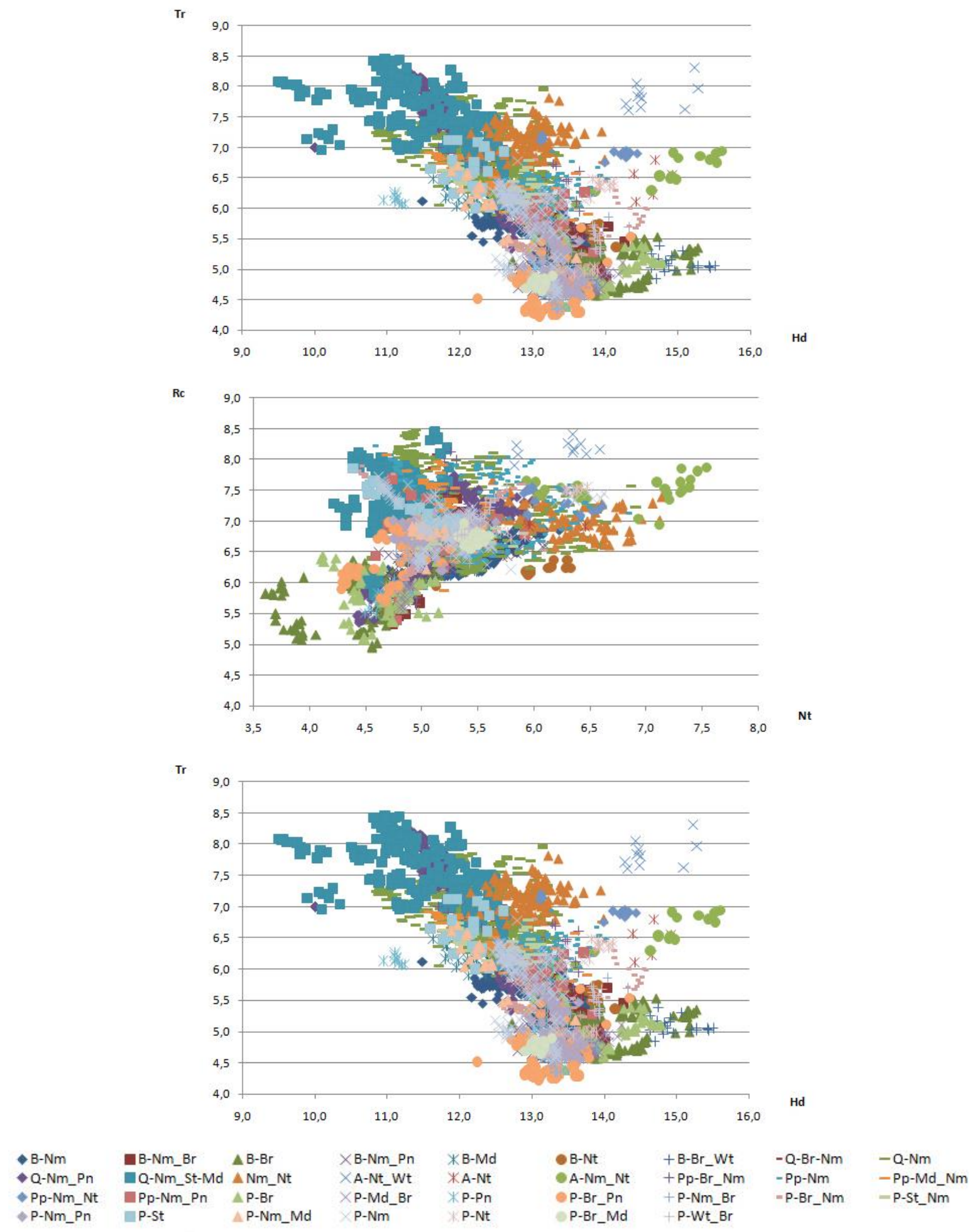

Fig. 2. Distribution of association groups of different formation communities in the ecological space.

Environmental factors: $\mathrm{Hd}$ - soil moisture content, $\mathrm{Nt}$ - soil nitrogen richness, $\mathrm{Rc}$ - soil acidity,

$\mathrm{Tr}$ - generalized salt regime of the soil, $\mathrm{Lc}$ - illumination - shading, $\mathrm{fH}$ - variability of soil moisture

In the shrub synusia, the greatest preservation rate of floristic diversity is noted in water-boreal aspen forests. The highest ontogenetic abundance is noted in communities with a high proportion of nemoral species (nemoral pine forests, broadleaved forests).

In the grass synusia, a low preservation rate of floristic diversity is noted in all types of forests, 
while a low participation of species of nemoral ECG is noted in all communities, except for nitrophilous-nemoral aspen forests.

All synusiae of pine forests, which cover the main part of the erosion-denudation plain landscapes, are floristically poor. The arboreal layer of these phytocenoses is often formed only by R-species, i.e. explents - Pinus sylvestris, Betula pendula и Populus tremula. Demographically complete populations of trees and shrubs are either absent in them, or their share is small. In the shrub synusia, the largest number of full-member populations is found in nemoral pine forests and only for shade-tolerant species (Euonymus verrucosa,
Corylus avellana). The grass layer is characterized by a low proportion of nemoral species.

Broad-leaved forests are "fragments" of polydominant broad-leaved and coniferous-broadleaved forests transformed by long-term economic activity. The share of participation of R-species (explents) in their stand is one of the lowest among all forest types in EDP landscapes. The degree of floristic richness is low in arboreal and shrub synusiae and very low in grass synusia. Demographically full-member populations are characteristic of a small number of shade-tolerant tree and shrub species (Acer platanoides, Tilia cordata, Euonymus verrucosa, Corylus avellana).

Table 5

Assessment of signs of the successional state of forests in EDP landscapes

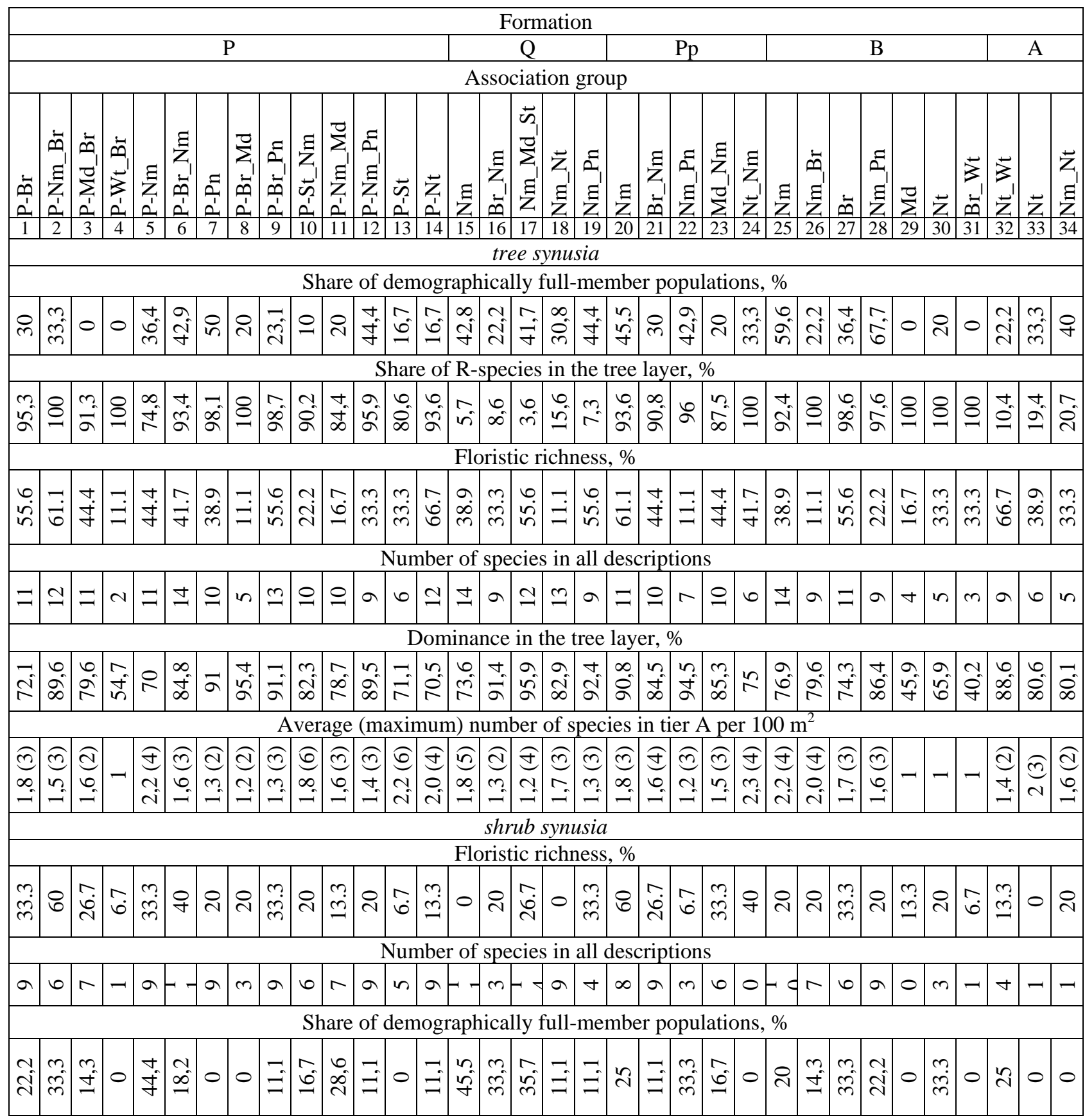


End of Table 5

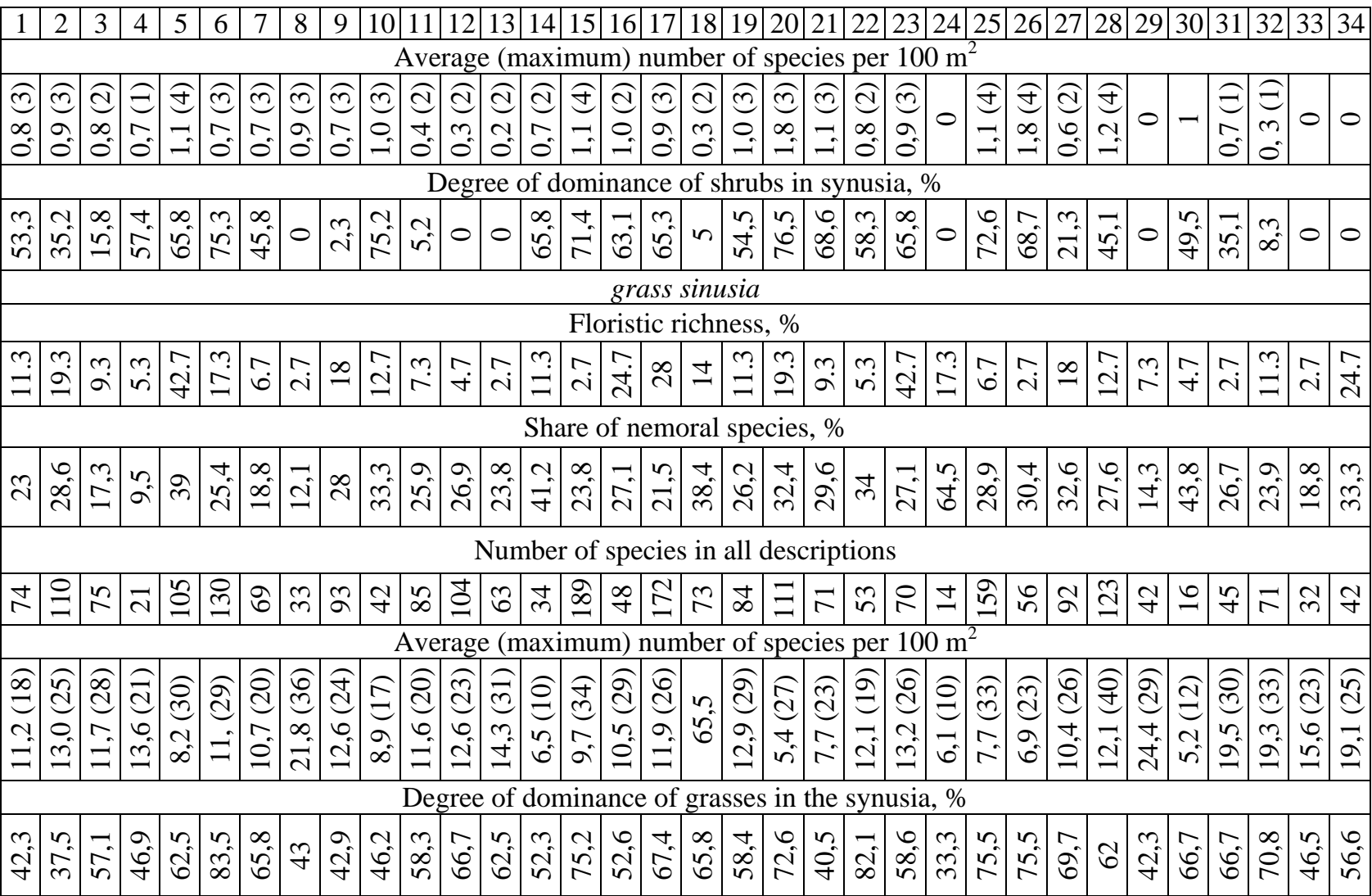
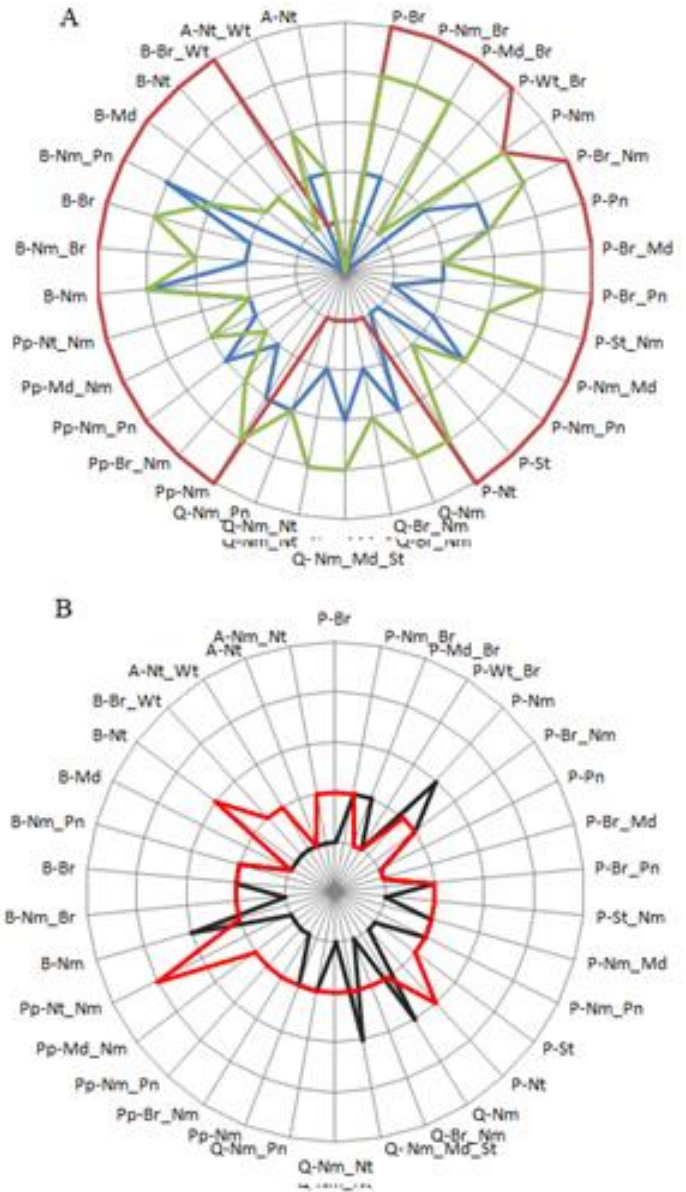

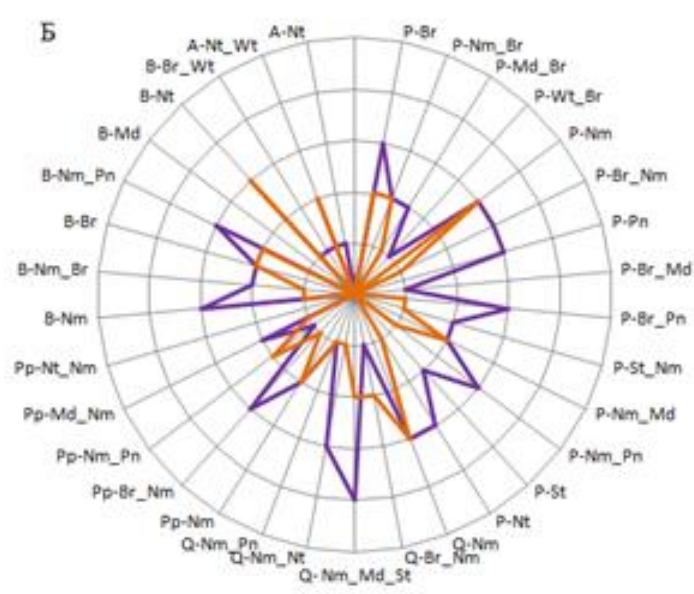

Signs of synusia

A - tree synusia:

- Floristic richness

__ Share of demographically full-member populations

- Share of R-species

B - shrub synusia:

_ Floristic richness Share of demographically full-member populations

B - grass sinusia:

- Floristic richness

- Share of nemoral species

Fig. 3. Assessment polygrams of the successional state of association groups of forest formations based on diagnostic features of different synusiae 
In the grass synusia, the share of potential flora is only $10-20 \%$, which is explained by the unfavorable light situation under the canopy of trees and shrubs, the absence of a gap mosaic, and only in deciduous nemoral and nemoral-meadow forests it is approximately $40 \%$. The participation share of species of nemoral ECG in the herbaceous cover is small.

Small-leaved forests (birch, aspen) are characterized by low values of floristic richness in all synusiae. In the arboreal layer, the explent species (R-species - Betula pendula, B. pubescens, Populus tremula) account for the greatest share. The largest share of demographically full-member tree populations is characteristic of nemoral and nemoral-upland birch forests, whereas nitrophilous birch forests are characterized by the largest share of shrubs. In the grass cover, the participation rate of nemoral ECG species is highest in nitrophilousnemoral aspen forests.
The black alder forests are characterized by the minimum scores of the successional state in all synusiae.

\section{Conclusion}

The assessment of the successional state showed that all modern forests are formed under the human influence and are transformed as a result of a complex combination of felling of different types and intensity, plowing, grazing, and fires.

The existing differences in the successional status of forests of different formations are manifested in the changing species composition of forest stands and floristic diversity of all community synusiae (trees, shrubs, grasses).

Anthropogenic activity always leads to depletion of the species composition, coevality of tree layers, and violation of the mosaic-layer organization of phytocenoses.

\section{Cnuсок numepamypы}

1. Ямашкин А. А., Артемова С. Н., Новикова Л. А. [и др.]. Ландшафтная карта и пространственные закономерности природной дифференциации Пензенской области // Проблемы региональной экологии. 2011. № 1. C. 49-57.

2. Артемова С. Н., Леонова Н. А. Морфологическая структура ландшафтов верхнего плато Приволжской возвышенности в пределах Пензенской области // Структурно-динамические особенности, современное состояние и проблемы оптимизации ландшафтов : материалы пятой Междунар. конф. Воронеж, 2013. С. 20-23.

3. Леонова Н. А., Кулакова Д. А., Артемова С. Н. Растительный покров ландшафтов верхнего плато Приволжской возвышенности в пределах Пензенской области // Известия высших учебных заведений. Поволжский регион. Естественные науки. 2013. № 1. С. 72-81.

4. Artemova S., Leonova N. Forest-steppe landscape organization of Eastern Europe (for example Penza region) // J. Wetlands Biodiversity. 2014. Vol. 4. P. 147-152.

5. Артемова С. Н., Леонова Н. А. Формирование ландшафтов северной лесостепи (на примере Пензенской области) // Фундаментальные исследования. 2014. № 11 (10). С. $2180-2184$.

6. Леонова Н. А., Артемова С. Н., Кулакова Д. А. Эколого-ценотическая структура фитоценозов верхнего плато Приволжской возвышенности в пределах Пензенской области // XXI век: итоги прошлого и проблемы настоящего плюс. 2013. № 09 (13), т. 1. С. 12-18.

7. Леонова Н. А. Пространственно-временная трансформация растительности верхнего плато Приволжской возвышенности (в пределах Пензенской области) // Фундаментальные исследования. 2014. № 9. С. 81-85.

8. Кулакова Д. А., Леонова Н. А. Приуроченность основных растительных сообществ к элементам рельефа в пределах верхнего плато Приволжской возвышенности // Материалы V Всероссийской геоботанической школы-конференции с международным участием (Санкт-Петербург, 4-9 октября 2015 г.). СПб., 2015. С. 98.

9. Леонова Н. А. История антропогенного преобразования растительного покрова лесостепных ландшафтов западных склонов Приволжской возвышенности // Известия высших учебных заведений. Поволжский регион. Естественные науки. 2016. № 4. С. 23-37.

10. Леонова Н. А. Бореальные сосняки эрозионно-денудационных равнин в границах Пензенской области // Известия высших учебных заведений. Поволжский регион. Естественные науки. 2019. № 4 (28). С. 126-141.

11. Оценка и сохранение биоразнообразия лесного покрова в заповедниках Европейской России / отв. ред. Л. Б. Заугольнова. М. : Научный мир, 2000. 196 с.

12. Смирнова О. В., Ханина Л. Г., Смирнов В. Э. Эколого-ценотические группы в растительном покрове лесного пояса Восточной Европы // Восточно-Европейские леса (история в голоцене и современность) / под ред. О. В. Смирновой. М. : Наука, 2004. Т. 1. С. 165-175.

13. European Russian Forests. Their Current State and Features of Their History / ed. by O. Smirnova, M. Bobrovsky, L. Khanina. Springer, 2017. Vol. 15. 566 p. Series Plant and Vegetation (Series editor Marinus J. A. Werger, Utrecht, The Netherlands). doi: 10.1007/978-94-024-1172-0

14. Смирнов В. Э., Ханина Л. Г., Бобровский М. В. Обоснование системы экологоценотических групп видов растений лесной зоны Европейской России на основе экологических шкал, геоботанических описаний и статистического анализа // Бюллетень Московского общества испытателей природы. Сер. биологическая. 2006. T. 111, № 2. C. 36-47. 
15. Ниценко А. А. Об изучении экологической структуры растительного покрова // Ботанический журнал. 1969. T. 54. $221 \mathrm{c.}$

16. Зозулин Г. М. Взаимоотношения лесной и травянистой растительности в Центрально-Черноземном гос. заповеднике // Труды Центр.-Чернозем. гос. заповедника. 1955. Вып. 3. С. 102-234.

17. Зозулин Г. М. Исторические свиты растительности европейской части СССР // Ботанический журнал. 1973. Т. 58, № 8. С. 1081-1092.

18. Смирнова О. В. Методологические подходы и методы оценки климаксового и сукцессионного состояния лесных экосистем (на примере Восточноевропейских лесов) // Лесоведение. 2004. № 3. С. 15-27.

19. Смирнова О. В., Бобровский М. В., Ханина Л. Г., Смирнов В. Э. Сукцессионный статус старовозрастных темнохвойных лесов Европейской России // Успехи современной биологии. 2006. Т. 126, № 1. С. $27-49$.

20. Сукцессионные процессы в заповедниках России и проблемы сохранения биологического разнообразия / под. ред. О. В. Смирновой, Е. С. Шапошникова. СПб. : РБО, 1999. 549 с.

21. Методические подходы к экологической оценке лесного покрова в бассейне малой реки / отв. ред. Л. Б. Заугольнова, Т. Ю. Браславская. М. : Товарищество научных изданий КМК, 2010. 383 с.

22. Цыганов Д. Н. Фитоиндикация экологических режимов в подзоне хвойно-широколиственных лесов. М. : Наука, 1983. 198 с.

\section{References}

1. Yamashkin A.A., Artemova S.N., Novikova L.A. [et al.]. Landscape map and spatial patterns natural differentiation the Penza region. Problemy regional'noy ekologii = Regional ecology problems. 2011;(1):49-57. (In Russ.)

2. Artemova S.N., Leonova N.A. Morphological structure of landscapes of the upper plateau of the Volga upland within the Penza region. Strukturno-dinamicheskie osobennosti, sovremennoe sostoyanie i problemy optimizatsii landshaftov: materialy pyatoy Mezhdunar. konf. = Structural and dynamic features, current state and problems of landscape optimization: materials of the fifth International conference. Voronezh, 2013:20-23. (In Russ.)

3. Leonova N.A., Kulakova D.A., Artemova S.N. The vegetation cover of the upper plateau of the Volga upland terrain within the Penza region. Izvestiya vysshikh uchebnykh zavedeniy. Povolzhskiy region. Estestvennye nauki = University proceedings. Volga region. Natural sciences. 2013;(1):72-81. (In Russ.)

4. Artemova S., Leonova N. Forest-steppe landscape organization of Eastern Europe (for example Penza region). J. Wetlands Biodiversity. 2014;4:147-152.

5. Artemova S.N., Leonova N.A. Formation of landscapes of the northern forest-steppe (on the example of the Penza region). Fundamental'nye issledovaniya = Fundamental research. 2014;(11):2180-2184. (In Russ.)

6. Leonova N.A., Artemova S.N., Kulakova D.A. Ecological-coenotic structure phytocenoses of the upper plateau of the Volga Upland within the Penza region. XXI vek: itogi proshlogo i problemy nastoyashchego plyus =XXI centure: resumes of the past and challenges of the present plus. 2013;(09):12-18. (In Russ.)

7. Leonova N.A. Spatial-temporal transformation of the vegetation upper plateau of the Volga Upland. Fundamental'nye issledovaniya = Fundamental research. 2014;(9):81-85. (In Russ.)

8. Kulakova D.A., Leonova N.A. The confinement of the main plant communities to the relief elements within the upper plateau of the Volga Upland. Materialy V Vserossiyskoy geobotanicheskoy shkoly-konferentsii s mezhdunarodnym uchastiem (Sankt-Peterburg, 4-9 oktyabrya 2015 g.) = Materials of the V All-Russian Geobotanical School-Conference with International Participation (St. Petersburg, October 4-9, 2015). Saint-Petersburg, 2015:98. (In Russ.)

9. Leonova N.A. The history of man-made transformation of vegetation on forest-steppe landscapes of western slopes of the Volga uplands. Izvestiya vysshikh uchebnykh zavedeniy. Povolzhskiy region. Estestvennye nauki = University proceedings. Volga region. Natural sciences. 2016;(4):23-37. (In Russ.)

10. Leonova N.A. Boreal pine forests of erosion-denudation plains within the boundaries of the Penza region. Izvestiya vysshikh uchebnykh zavedeniy. Povolzhskiy region. Estestvennye nauki = University proceedings. Volga region. Natural sciences. 2019;(4):126-141. (In Russ.)

11. Zaugol'nova L.B. [ed.]. Otsenka i sokhranenie bioraznoobraziya lesnogo pokrova v zapovednikakh Evropeyskoy Rossii = Assessment and conservation of forest biodiversity in the reserves of European Russia. Moscow: Nauchnyy mir, 2000:196. (In Russ.)

12. Smirnova O.V., Khanina L.G., Smirnov V.E. Ecological- coenotical groups in the vegetation cover of the forest belt of Eastern Europe. Vostochno-Evropeyskie lesa (istoriya v golotsene $i$ sovremennost') = East European forests: history in the Holocene and modern times. Moscow: Nauka, 2004;1:165-175. (In Russ.)

13. Smirnova O., Bobrovsky M., Khanina L. [eds.]. European Russian Forests. Their Current State and Features of Their History. Springer, 2017;15:566. Series Plant and Vegetation (Series editor Marinus J. A. Werger, Utrecht, The Netherlands). doi: 10.1007/978-94-024-1172-0

14. Smirnov V.E., Khanina L.G., Bobrovskiy M.V. Validation of the ecological-coenotical groups of vascular plant species for european russian forests on the basis of ecological indicator values, vegetation releves and statistical analysis. Byulleten' Moskovskogo obshchestva ispytateley prirody. Ser. Biologicheskaya = Bulletin of Moscow Society of Naturalists/ Biological series. 2006;111(2):36-47. (In Russ.) 
15. Nitsenko A.A. On the study of the ecological structure of the vegetation cover. Botanicheskiy zhurnal = Botanical journal. 1969;54:221. (In Russ.)

16. Zozulin G.M. The relationship of forest and herbaceous vegetation in the Central Black Earth State Reserve. Trudy Tsentr.-Chernozem. gos. Zapovednika = Proceedings of the Central Black Earth State Reserve. 1955;(3):102-234. (In Russ.)

17. Zozulin G.M. Historical suite of vegetation of the European part of the USSR. Botanicheskiy zhurnal = Botanical journal. 1973;58(8):1081-1092. (In Russ.)

18. Smirnova O.V. Methodological approaches and methods of assessing climax and succession state of forest ecosystems (from the example of East European forests). Lesovedenie = Forestry. 2004;(3):15-27. (In Russ.)

19. Smirnova O.V., Bobrovskiy M.V., Khanina L.G., Smirnov V.E. Succession status of old-growth spruce and spruce-fir forests in European Russia. Uspekhi sovremennoy biologii = Successes of modern biology. 2006; 126(1):27-49. (In Russ.)

20. Smirnova O.V., Shaposhnikov E.S. [eds.]. Suktsessionnye protsessy v zapovednikakh Rossii $i$ problemy sokhraneniya biologicheskogo raznoobraziya = Successional Processes in Reserves of Russia and Problems of Biodiversity Conservation. Saint-Petersburg: RBO, 1999:549. (In Russ.)

21. Zaugol'nova L. B., Braslavskaya T. Yu. [ed.]. Metodicheskie podkhody k ekologicheskoy otsenke lesnogo pokrova $v$ basseyne maloy reki $=$ Methodological approaches to the ecological assessment of forest cover in the basin of a small river. Moscow: Tovarishchestvo nauchnykh izdaniy KMK, 2010:383. (In Russ.)

22. Tsyganov D.N. Fitoindikatsiya ekologicheskikh rezhimov v podzone khvoyno-shirokolistvennykh lesov = Phytoindication of ecological regimes in the subzone of coniferous-deciduous forests. Moscow: Nauka, 1983:198. (In Russ.) 